\title{
The psychometric properties of the St George's Respiratory Questionnaire (SGRQ) in patients with idiopathic pulmonary fibrosis: a literature review
}

\author{
Jeffrey J Swigris ${ }^{1 *}$, Dirk Esser ${ }^{2}$, Craig S Conoscenti ${ }^{3}$ and Kevin K Brown ${ }^{1}$
}

\begin{abstract}
Assessment of health-related quality of life (HRQL) is particularly important in patients with progressive and incurable diseases such as idiopathic pulmonary fibrosis (IPF). The St George's Respiratory Questionnaire (SGRQ) has frequently been used to measure HRQL in patients with IPF, but it was developed for patients with obstructive lung diseases. The aim of this review was to examine published data on the psychometric performance of the SGRQ in patients with IPF. A comprehensive search was conducted to identify studies reporting data on the internal consistency, construct validity, test-retest reliability, and interpretability of the SGRQ in patients with IPF, published up to August 2013. In total, data from 30 papers were reviewed. Internal consistency was moderate for the SGRQ symptoms score and excellent for the SGRQ activity, impact and total scores. Validity of the SGRQ symptoms, activity, impact and total scores was supported by moderate to strong correlations with other patient-reported outcome measures and with a measure of exercise capacity. Most correlations were moderately strong between SGRQ activity or total scores and forced or static vital capacity, the most commonly used marker of IPF severity. There was evidence that changes in SGRQ domain and total scores could detect within-subject improvement in health status, and differentiate groups of patients whose health status had improved, declined or remained unchanged. Although the SGRQ was not developed specifically for use with patients with IPF, on balance, its psychometric properties are adequate and suggest that it may be a useful measure of HRQL in this patient population. However, several questions remain unaddressed, and further research is needed to confirm the SGRQ's utility in IPF.
\end{abstract}

Keywords: Idiopathic pulmonary fibrosis, Patient-reported outcomes, PROs, St George's Respiratory Questionnaire, SGRQ, Health-related quality of life, HRQL, Psychometrics, Validity, Reliability

\section{Introduction}

Idiopathic pulmonary fibrosis (IPF) is a specific form of fibrosing interstitial pneumonia characterized by progressive worsening of dyspnea and lung function [1]. In the United States, the annual incidence of IPF has been estimated as 6.8-8.8 cases per 100,000 using narrow case definitions (requiring a definite pattern of Usual Interstitial Pneumonia [UIP] on high-resolution computed tomography [HRCT]), and as 16.3-17.4 cases per 100,000 using broad case definitions (including patients with a possible UIP-pattern on HRCT) [2]. Although IPF

\footnotetext{
* Correspondence: swigris」@NJHealth.org

${ }^{1}$ National Jewish Health, Denver, CO, USA

Full list of author information is available at the end of the article
}

has a poor prognosis, with a median survival time from diagnosis of 2 to 3 years, the clinical course of IPF varies considerably $[1,3]$. Symptoms experienced by patients with IPF include non-productive cough, fatigue and chronic dyspnea, with the latter being the most prominent and disabling [4]. The morbidity associated with IPF has a broad and profound impact on patients' healthrelated quality of life (HRQL) $[4,5]$.

As IPF is a progressive disease with no cure, HRQL and other patient-centered outcomes are important endpoints to evaluate in research and clinical practice [6]. Although no disease-specific measure of HRQL has been established as suitable for longitudinal research in patients with IPF, several HRQL instruments (and others, including symptom and generic quality of life questionnaires) have been used $[7,8]$. Which patient-centered 
instrument(s) (including HRQL questionnaires) to use in a particular study depends on a number of factors, including the design of the study, the intervention being assessed, the hypotheses being tested, and the characteristics of the comparator group (general population, patients with IPF of different severity, patients with another disease, etc.). In any situation, whether a generic HRQL instrument might perform as well or better than a disease-specific HRQL instrument is uncertain.

In this review, we focused on the St George's Respiratory Questionnaire (SGRQ). Although originally developed for use in patients with chronic obstructive pulmonary disease (COPD) and asthma [8], it has frequently been used to evaluate HRQL in patients with IPF. The SGRQ is a 50item questionnaire split into three domains: symptoms (assessing the frequency and severity of respiratory symptoms), activity (assessing the effects of breathlessness on mobility and physical activity), and impact (assessing the psychosocial impact of the disease) [9]. Scores are weighted such that every domain score and the total score range from 0 to 100 , with higher scores indicating a poorer HRQL.

The aim of this review was to assess the appropriateness of the SGRQ for measuring HRQL in patients with IPF by examining the evidence relating to the psychometric performance of the SGRQ in this population. A revised version of the SGRQ, the SGRQ-I, has been developed for use in patients with IPF [10]; however, studies assessing this tool are limited, and SGRQ-I data are not covered in this manuscript.

\section{Methods}

\section{Search strategy and data extraction}

A comprehensive literature review was conducted to identify articles that evaluated the psychometric properties of the SGRQ in patients with IPF. Following a PubMed search (see Additional file 1), articles were excluded if they were not published between 1 January 1991 (date of first publication of the SGRQ) and 31 August 2013, were not published in English, did not report data on the psychometric properties of the SGRQ in patients with IPF or duplicated clinical trial data reported in another article (Figure 1). Data extracted from the studies included study characteristics (country, duration, design, sample size), participant characteristics (age, gender, time since diagnosis, forced vital capacity $[$ FVC]\% predicted, diffusing capacity for carbon monoxide $\left[\mathrm{DL}_{\mathrm{CO}}\right] \%$ predicted) and results of the psychometric tests.

Articles were selected that assessed any of the following psychometric properties of the SGRQ: internal consistency, convergent validity, known groups validity, test-retest reliability (reproducibility), responsiveness, minimal important difference (MID), and floor and ceiling effects [11]. Internal consistency refers to the degree to which the individual items within an instrument correlate with each other (i.e., tap the same underlying construct). This is determined using Cronbach's coefficient alpha, with $\geq 0.70$ considered to indicate acceptable internal consistency for a multi-dimensional instrument. Convergent validity describes the degree to which two

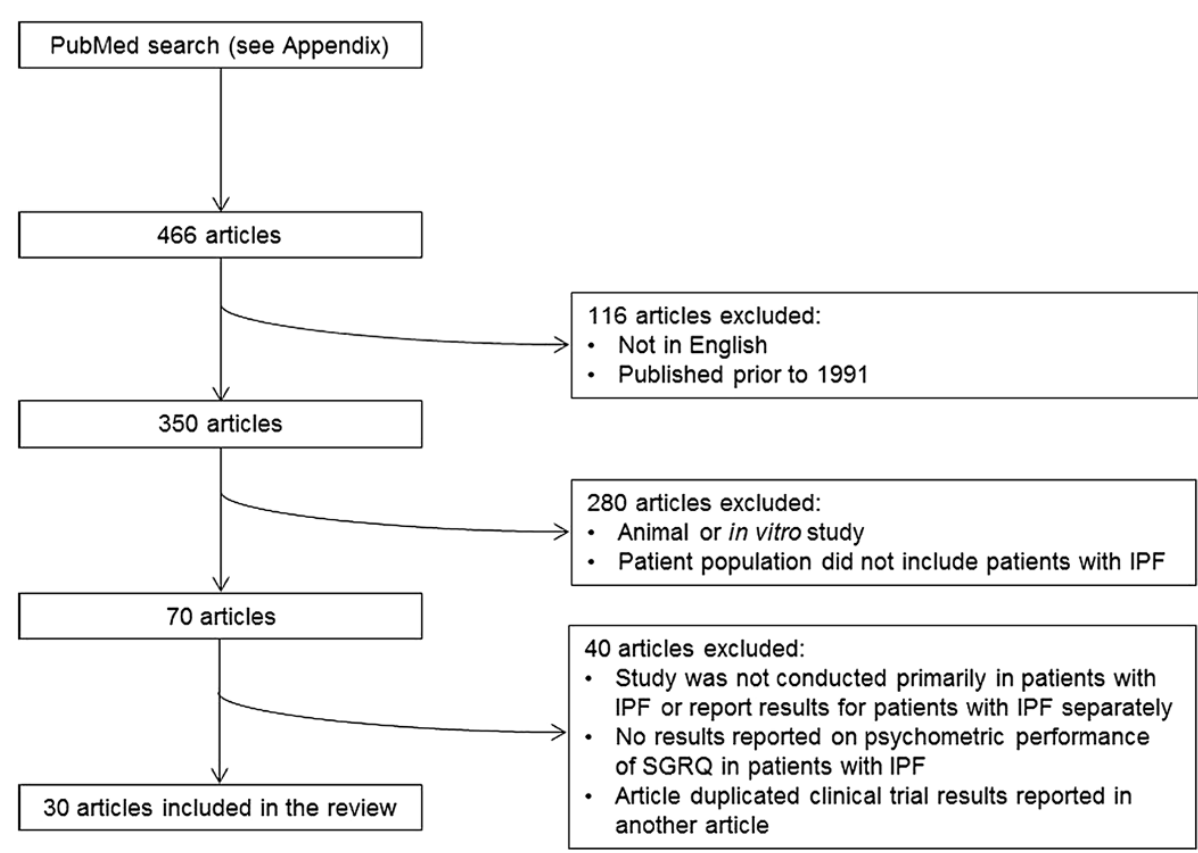

Figure 1 Selection of articles to be included in the review. 
measures, hypothesized to measure the same construct, correlate. Known groups validity refers to the extent to which scores on an instrument distinguish groups that differ on a key variable, usually clinical in nature. For the described validity measures, correlations were regarded as weak if $\leq 0.30$, moderate if $0.30-0.60$, and strong if $>0.60$ [12]. Test-retest reliability assesses the ability of an instrument to produce consistent scores over repeated measurements in patients who are clinically stable. Responsiveness assesses the ability of an instrument to detect change in individuals who are hypothesized to have changed on the underlying construct (HRQL) and who are known to have experienced change in clinical status. MID estimates identify the smallest difference in the score on an instrument that patients perceive as important. Floor and ceiling effects are limitations that occur when an individual scores at the extremes of an instrument; if a patient's score is the lowest or highest possible value, the instrument is unable to detect a reduction or increase, respectively.

\section{Results}

A total of 30 papers were included in the review (Figure 1; Table 1).

\section{Internal consistency}

Data from a clinical trial of bosentan have been used to determine the internal consistency of the SGRQ in patients with IPF. Cronbach's alpha was 0.66 for the symptoms score and $\geq 0.84$ for each of the SGRQ activity, impact and total scores $[10,34]$.

\section{Convergent validity}

Convergent validity was evaluated by extracting crosssectional and longitudinal correlations between SGRQ scores and other patient-reported outcome measures (Table 2), an assessment of exercise capacity (Table 3), pulmonary function tests (PFTs) or partial pressure of arterial oxygen (Table 4), and assessments of fibrotic abnormalities on HRCT (Table 5).

\section{Patient-reported outcome measures}

In nine studies, investigators provided information on the correlation between SGRQ scores and other patientreported outcome measures (BDI [Baseline Dyspnea Index], D-12 [Dyspnea-12], K-BILD [King's Brief Interstitial Lung Disease questionnaire], UCSD-SOBQ [University of California San Diego Shortness of Breath Questionnaire], CQLQ [Cough Quality of Life Questionnaire], a single-item dyspnea assessment, SF-36 Physical Component Summary score [SF-36 PCS] and the Borg Dyspnea Index) (Table 2). Moderate to strong correlations were observed between the SGRQ total score and the total scores on these instruments (Table 2). In general, moderate to strong correlations were observed between SGRQ domain scores and the total scores on these instruments. Likewise, moderate to strong correlations were observed between SGRQ domain or total scores and the total, physical complaints, extreme physical complaints, and functional ability sub-scale scores of the CQLQ ( $r=0.34$ to 0.81 ) [21], the total and sub-scale scores of the K-BILD $(r=-0.59$ to -0.89) [27], the SF-36 PCS, a composite score measuring overall physical health $(\mathrm{r}=-0.52$ to -0.74$)[10]$ and the Borg Dyspnea index ( $r=0.35$ to 0.56$)[10,15]$. For most measures and their sub-scales, correlations were weakest with the SGRQ symptoms score (when compared with other SGRQ domains or the total score).

In two studies, investigators evaluated correlations between SGRQ change scores and change scores from other patient-reported outcome measures (Table 2). In one study, correlations were moderately strong between change scores for the SGRQ activity, impact and total scores and change scores from the single-item dyspnea assessment ( $r=0.59,0.56$ and 0.45 , respectively) [28]. In the other study, investigators found that the correlation between the BDI change score and SGRQ total change score was -0.29 and not significant [25]. However, the BDI was designed to measure dyspnea severity at a single point in time and not to measure change in dyspnea severity [42].

\section{Measures of exercise capacity}

Correlation coefficients between SGRQ scores and a measure of exercise capacity are presented in Table 3. Distance covered during the 6 -minute walk test $(6 \mathrm{MWD})$ is frequently used as a measure of exercise capacity in patients with IPF, and change in 6MWD has been shown to be a predictor of mortality in these patients [16]. In five cross-sectional studies in patients with IPF, investigators examined the relationship between the SGRQ total score and 6MWD. The strength of these correlations was moderate to strong in three $(-0.45$ to -0.72$)[15,28,40]$ and weak in two $(-0.26$ and -0.28$)[10,16]$ studies. In four cross-sectional studies, investigators examined the relationship between the SGRQ domain scores and the 6MWD $[10,28,39,40]$; the strength of these correlations was moderate to strong for the activity score in all four studies $(r=-0.32$ to -0.72$)$, moderate to strong for the impact score $(r=-0.41$ to -0.63$)$ and moderate for the symptoms score $(r=-0.32$ to -0.41$)$ in three studies. In three studies, investigators examined the relationship between change scores for the SGRQ total and change in 6MWD; $[16,25,28]$ correlation coefficients ranged from -0.23 to -0.43 .

\section{Pulmonary function tests and arterial blood gas analysis}

Table 4 presents correlations between SGRQ scores and either PFTs or arterial blood gas analysis in patients with IPF. All correlations between the SGRQ total score and these variables were moderate to strong $(r=-0.30$ 
Table 1 Studies included in this review

\begin{tabular}{|c|c|c|c|c|c|c|c|c|c|}
\hline \multirow[t]{2}{*}{ Study } & \multirow[t]{2}{*}{ Study type } & \multirow[t]{2}{*}{$\begin{array}{l}\text { Experimental } \\
\text { treatment }\end{array}$} & \multirow[t]{2}{*}{ Country } & \multicolumn{2}{|c|}{$\begin{array}{l}\text { Sample } \\
\text { size }^{1}\end{array}$} & \multirow[t]{2}{*}{$\begin{array}{l}\text { Disease duration } \\
\text { (mo) mean (SD) }\end{array}$} & \multirow[t]{2}{*}{ Baseline SGRQ score $^{2}$} & \multicolumn{2}{|c|}{ Baseline spirometric values $^{2}$} \\
\hline & & & & All & IPF & & & FVC\% predicted & $\begin{array}{l}\mathrm{DL}_{\mathrm{co}} \% \\
\text { predicted }\end{array}$ \\
\hline $\begin{array}{l}\text { Antoniou et al., } \\
2006 \text { [13] }\end{array}$ & $\mathrm{RCT}$ & $\begin{array}{l}\text { Interferon } \\
\text { gamma b }\end{array}$ & Greece & 50 & 50 & $\begin{array}{l}T=49.4(24.3) \\
C=42.7(16.8)\end{array}$ & - & $\begin{array}{l}T=71.8(15.0) \\
C=70.7(17.7)\end{array}$ & - \\
\hline $\begin{array}{l}\text { Berry et al., } \\
2012 \text { [14] }\end{array}$ & $\begin{array}{l}\text { Secondary } \\
\text { validation study }\end{array}$ & $\mathrm{n} / \mathrm{a}$ & US & 405 & 239 & - & - & $66.0(52-78)$ & - \\
\hline $\begin{array}{l}\text { Chang et al., } \\
1999 \text { [15] }\end{array}$ & $\begin{array}{l}\text { Standalone } \\
\text { validation study }\end{array}$ & $\mathrm{n} / \mathrm{a}$ & US & 50 & 33 & - & $\begin{array}{l}\text { Total }=38.9[28.7-55.2] \\
\text { Symptoms domain }=50.5[31.5-69.8] \\
\text { Activity domain }=54.4[39.9-72.9] \\
\text { Impact domain = } 28.4[18.8-45.1]\end{array}$ & $65.0(49.0-81.0)$ & $49.0(36.5-59.8)$ \\
\hline $\begin{array}{l}\text { du Bois et al., } \\
2011 \text { [16] }\end{array}$ & $\mathrm{RCT}$ & $\begin{array}{l}\text { Interferon } \\
\text { gamma b }\end{array}$ & Multi-national & 822 & 822 & - & Total $=41.8(18)$ & $72.5(12.7)$ & $47.4(9.2)$ \\
\hline $\begin{array}{l}\text { Han et al., } \\
2013 \text { [17] }\end{array}$ & $\mathrm{RCT}$ & Sildenafil & US & 119 & 119 & 20.4 & - & 56.9 & 26.0 \\
\hline $\begin{array}{l}\text { Horton et al., } \\
2012 \text { [18] }\end{array}$ & $\mathrm{RCT}$ & Thalidomide & US & 23 & 23 & $20.5(3-59)$ & $\begin{array}{l}\text { Total }=57.4(18.8) \\
\text { Symptoms domain }=67.7(19.7), \\
\text { Activity domain }=64.3(22.7) \\
\text { Impact domain = 48.1 }\end{array}$ & $70.4(13.7)$ & $57.4(14.4)$ \\
\hline $\begin{array}{l}\text { King, Jr. et al., } \\
2008 \text { [19] }\end{array}$ & $\mathrm{RCT}$ & Bosentan & Multi-national & 158 & 158 & $\begin{array}{l}T=12.2(12.2) \\
C=12.1(12.0)\end{array}$ & T-Total $=45.7(18.1), C-$ Total $=45.2(19)$ & $\begin{array}{l}T=65.9(10.5), \\
C=69.5(12.6)\end{array}$ & $\begin{array}{l}\mathrm{T}=42.3(9.5) \\
\mathrm{C}=41.4(9.5)\end{array}$ \\
\hline $\begin{array}{l}\text { King, Jr. et al., } \\
2009 \text { [20] }\end{array}$ & $\mathrm{RCT}$ & $\begin{array}{l}\text { Interferon } \\
\text { gamma b }\end{array}$ & Multi-national & 826 & 826 & - & T-Total = 41.6 (17.9), C-Total = $42.4(18.2)$ & $\begin{array}{l}T=72.2(12.3) \\
C=73.1(13.4)\end{array}$ & $\begin{array}{l}\mathrm{T}=47.4(9.2) \\
\mathrm{C}=47.3(9.3)\end{array}$ \\
\hline $\begin{array}{l}\text { Lechtzin et al., } \\
2013 \text { [21] }\end{array}$ & $\mathrm{RCT}$ & Thalidomide & US & 24 & 24 & - & $\begin{array}{l}\text { Total }=57.4(18.8) \\
\text { Symptoms domain }=67.7(19.7), \\
\text { Activity domain }=64.3(22.7) \\
\text { Impact domain = 48.1 }\end{array}$ & $70.4(13.7)$ & $57.4(14.4)$ \\
\hline $\begin{array}{l}\text { Mishra et al., } \\
2011 \text { [22] }\end{array}$ & Within-subject trial & Oral doxycycline & India & 6 & 6 & - & Total = $50.90(8.38)$ & $\mathrm{n} / \mathrm{a}$ & $n / a$ \\
\hline $\begin{array}{l}\text { Naji et al., } \\
2006 \text { [23] }\end{array}$ & Within-subject trial & $\begin{array}{l}\text { Pulmonary } \\
\text { rehabilitation }\end{array}$ & Ireland & 26 & 19 & - & Total $=48(27.6,67.9)$ & $66.7(20.7)$ & $42.5(14)$ \\
\hline $\begin{array}{l}\text { Nishiyama et al., } \\
2005 \text { [24] }\end{array}$ & $\begin{array}{l}\text { Standalone } \\
\text { validation study }\end{array}$ & $\mathrm{n} / \mathrm{a}$ & Japan & 41 & 41 & - & $\begin{array}{l}\text { Total }=35.7 \text { (20.6) [range 1.6-77.6], } \\
\text { Symptoms domain = 40.1 (24.6) } \\
\text { [range 4.4-85.6], Activity domain }=44.5 \\
(26.7) \text { [range 0-93.9], Impact domain }=28.9 \\
\text { (19.8) [range 0-77.0] }\end{array}$ & $\mathrm{n} / \mathrm{a}$ & $n / a$ \\
\hline
\end{tabular}


Table 1 Studies included in this review (Continued)

\begin{tabular}{|c|c|c|c|c|c|c|c|c|c|}
\hline $\begin{array}{l}\text { Nishiyama et al., } \\
2008 \text { [25] }\end{array}$ & $\mathrm{RCT}$ & $\begin{array}{l}\text { Pulmonary } \\
\text { rehabilitation }\end{array}$ & Japan & 28 & 28 & - & $\begin{array}{l}\text { T-Total = } 50.2(16.3), \\
\text { T-Symptoms domain }=56.4(22.3), \\
\text { T-Activity domain = } 64.7(17.1), \\
\text { T-Impact domain }=39.7(17.6), \\
\text { C-Total = } 37.8(22.7) \\
\text { C-Symptoms domain }=38.0(25.8), \\
\text { C-Activity domain = } 50.4(26.2), \\
\text { C-Impact domain }=29.9(23.7)\end{array}$ & $\begin{array}{l}T=66.1(13.2) \\
C=68.7(19.5)\end{array}$ & $\begin{array}{l}T=59.4(16.7) \\
C=48.6(16.7)\end{array}$ \\
\hline $\begin{array}{l}\text { Noth et al., } \\
2012 \text { [26] }\end{array}$ & $\mathrm{RCT}$ & Warfarin & US & 145 & 145 & $\mathrm{~T}=21.6, \mathrm{C}=25.2$ & T-Total $=46.2(18.0), C-$ Total $=50.1$ (17.2) & $\begin{array}{l}T=58.9(16.2) \\
C=58.7(16.1)\end{array}$ & $\begin{array}{l}T=33.8(12.4) \\
C=34.6(13.4)\end{array}$ \\
\hline $\begin{array}{l}\text { Patel et al., } \\
2012 \text { [27] }\end{array}$ & $\begin{array}{l}\text { Standalone } \\
\text { validation study }\end{array}$ & $\mathrm{n} / \mathrm{a}$ & UK & 173 & 49 & 48.0 & - & $82(34-143)$ & - \\
\hline $\begin{array}{l}\text { Peng et al., } \\
2008 \text { [28] }\end{array}$ & $\begin{array}{l}\text { Standalone } \\
\text { validation study }\end{array}$ & $\mathrm{n} / \mathrm{a}$ & China & 68 & 68 & $14.0(14.0)$ & $\begin{array}{l}\text { Total = } 54(15) \\
\text { Symptoms domain = } 65(16), \\
\text { Activity domain = } 56(15) \\
\text { Impact domain = } 49(19)\end{array}$ & $66(18)$ & $54(16)$ \\
\hline $\begin{array}{l}\text { Raghu et al., } \\
2004 \text { [29] }\end{array}$ & $\mathrm{RCT}$ & $\begin{array}{l}\text { Interferon } \\
\text { gamma b }\end{array}$ & Multi-national & 330 & 330 & - & - & $\begin{array}{l}\mathrm{T}=63.9(10.7) \\
\mathrm{C}=64.1(11.3)\end{array}$ & - \\
\hline $\begin{array}{l}\text { Raghu et al., } \\
2008 \text { [30] }\end{array}$ & $\mathrm{RCT}$ & Etanercept & Multi-national & 88 & 88 & $\begin{array}{l}T=14.7(19.8) \\
C=12.3(13.6)\end{array}$ & T-Total $=40.8(18.1), C-$ Total $=42.9(19.4)$ & $\begin{array}{l}T=64.7(14.1) \\
C=63.0(12.7)\end{array}$ & $\begin{array}{l}T=36.3(12.6) \\
C=36.9(10.8)\end{array}$ \\
\hline $\begin{array}{l}\text { Raghu et al., } \\
2013 \text { [31] }\end{array}$ & $\mathrm{RCT}$ & Ambrisentan & Multi-national & 492 & 492 & $T=13.2, C=10.8$ & T-Total $=44.5$ (21.6), C-Total = $40.5(21.1)$ & $\begin{array}{l}T=68.7(13.1) \\
C=69.9(13.8)\end{array}$ & $\begin{array}{l}T=42.0(13.8) \\
C=45.6(13.3)\end{array}$ \\
\hline $\begin{array}{l}\text { Rammaert et al., } \\
2009 \text { [32] }\end{array}$ & Within-subject trial & $\begin{array}{l}\text { Pulmonary } \\
\text { rehabilitation }\end{array}$ & France & 13 & 13 & - & - & $67(14)$ & $32(13)$ \\
\hline $\begin{array}{l}\text { Richeldi et al., } \\
2011 \text { [33] }\end{array}$ & $\mathrm{RCT}$ & $\begin{array}{l}\text { Nintedanib } \\
\text { (BIBF 1120) }\end{array}$ & Multi-national & 428 & 428 & $\begin{array}{l}\mathrm{T}, 50 \mathrm{mg} \mathrm{qd}=16.8 \\
(15.6), \mathrm{T}, 50 \mathrm{mg} \\
\mathrm{bid}=13.2(14.4), \\
\mathrm{T}, 100 \mathrm{mg} \mathrm{bid}=14.4 \\
(14.4), \mathrm{T}, 150 \mathrm{mg} \\
\mathrm{bid}=12(14.4), \\
\mathrm{C}=16.8(18)\end{array}$ & $\begin{array}{l}\text { T, } 50 \mathrm{mg} \text { qd-Total }=43.7(17.5) \\
\text { T, } 50 \mathrm{mg} \text { bid-Total }=42.5(17.0) \\
\text { T, } 100 \mathrm{mg} \text { bid-Total }=43.7(16.6) \\
\text { T, } 150 \mathrm{mg} \text { bid-Total }=40.1(18.3) \\
\text { C-Total = }\end{array}$ & $\begin{array}{l}\text { T, } 50 \mathrm{mg} \mathrm{qd}=80.4 \\
(17.8), \mathrm{T}, 50 \mathrm{mg} \mathrm{bid}=79.8 \\
(15.8), \mathrm{T}, 100 \mathrm{mg} \mathrm{bid}=85.5 \\
(19.2), \mathrm{T}, 150 \mathrm{mg} \mathrm{bid}=79.1 \\
(18.5), \mathrm{C}=81.7(17.6)\end{array}$ & - \\
\hline $\begin{array}{l}\text { Swigris et al., } \\
2010 \text { [34] }\end{array}$ & $\begin{array}{l}\text { Secondary } \\
\text { validation study }\end{array}$ & Bosentan & Multi-national & 158 & 158 & - & $\begin{array}{l}\text { Total }=44.8(19.5) \\
\text { Symptoms domain }=50.1(21.9), \\
\text { Activity domain }=60.6(22.8), \\
\text { Impact domain = } 33.7(20.6)\end{array}$ & $67.0(12.8)$ & $40.98(10.1)$ \\
\hline $\begin{array}{l}\text { Swigris et al., } \\
2012 \text { [35] }\end{array}$ & $\begin{array}{l}\text { Secondary } \\
\text { validation study }\end{array}$ & Sildenafil & US & 180 & 180 & 24.0 & Activity domain = $69.6(17.6)$ & $56.8(14.2)$ & $26.3(6.1)$ \\
\hline $\begin{array}{l}\text { Tzanakis et al., } \\
2005 \text { [36] }\end{array}$ & $\begin{array}{l}\text { Standalone } \\
\text { validation study }\end{array}$ & $\mathrm{n} / \mathrm{a}$ & Greece & 25 & 25 & 31.2 & $\begin{array}{l}\text { Total = } 37.7(18.9) \\
\text { Symptoms domain }=55.9(25.3) \text {, } \\
\text { Activity domain }=36.2(21.4) \\
\text { Impact domain = } 29.6(21)\end{array}$ & $68.8(16)$ & - \\
\hline \multirow[t]{2}{*}{$\begin{array}{l}\text { Tzouvelekis et al., } \\
2013 \text { [37] }\end{array}$} & Within-subject trial & $\begin{array}{l}\text { Adipose-derived } \\
\text { stromal cells }\end{array}$ & Greece & 14 & 14 & - & - & - & - \\
\hline & & $\mathrm{n} / \mathrm{a}$ & Canada & 137 & 137 & - & & $61.7(19.8)$ & 49.5 (17.9) \\
\hline
\end{tabular}


Table 1 Studies included in this review (Continued)

\section{Verma et al., Standalone}

2011 [38] $\quad$ validation study

\begin{tabular}{|c|c|c|c|c|c|}
\hline $\begin{array}{l}\text { Yorke et al., } \\
2010^{3} \text { [10] }\end{array}$ & $\begin{array}{l}\text { Secondary } \\
\text { validation study }\end{array}$ & Bosentan & Multi-national & 158 & 158 \\
\hline $\begin{array}{l}\text { Yorke et al., } \\
2011 \text { [39] }\end{array}$ & $\begin{array}{l}\text { Standalone } \\
\text { validation study }\end{array}$ & $\mathrm{n} / \mathrm{a}$ & Multi-national & 101 & 67 \\
\hline
\end{tabular}

validation study

Zimmermann et al., Standalone

2007 [40] validation study

$\mathrm{n} / \mathrm{a}$

$\mathrm{n} / \mathrm{a}$

Brazil

22

Zisman et al.,

$\mathrm{RCT}$

Sildenafil

US

$180180 \quad \mathrm{~T}=24.4, \mathrm{C}=22.4$

$2010[41]$

${ }^{1}$ Sample size reported represents the population in which efficacy was assessed. RCT = randomized controlled trial; $\mathrm{T}=$ treatment group; $\mathrm{C}=$ comparator group; qd = once daily; bid $=$ twice daily. ${ }^{2}$ Mean (SD) or

median [interquartile range] are reported based on availability. ${ }^{3}$ Data reported refer to the original version of the SGRQ, not the SGRQ-I.

Total $=63.4(3.7-96.3)$,

Symptoms domain $=59.8(0-97.2)$,

Activity domain $=81.6(6.0-99.5)$,

Impact domain $=54.1(0-96.4)$$$
-
$$

Total $=53(24)$,

Symptoms domain $=61$ (23),

Activity domain $=65$ (30)

Impact domain = $41(24)$

Total = 48.4 (17.9),

$70.4(19.4)$

Symptoms domain $=46.4(20.3)$,

(1.5

Activity domain $=62.4(19)$,

Impact domain $=43.6(20.9)$ 
Table 2 Correlation coefficients between SGRQ scores and other patient-reported assessments of health status

\begin{tabular}{|c|c|c|c|c|c|c|}
\hline & Measure & Scale & $\begin{array}{l}\text { Correlation } \\
\text { with SGRQ } \\
\text { symptoms } \\
\text { domain score }\end{array}$ & $\begin{array}{l}\text { Correlation } \\
\text { with SGRQ } \\
\text { activity domain } \\
\text { score }\end{array}$ & $\begin{array}{l}\text { Correlation } \\
\text { with SGRQ } \\
\text { impact domain } \\
\text { score }\end{array}$ & $\begin{array}{l}\text { Correlation } \\
\text { with SGRQ } \\
\text { total score }\end{array}$ \\
\hline \multicolumn{7}{|l|}{ Cross-sectional studies } \\
\hline Chang et al., 1999 [15] & Borg Dyspnea Index & & & & & $0.56^{\dagger}$ \\
\hline \multirow[t]{7}{*}{ Lechtzin et al., 2013 [21] } & CQLQ & Total & $0.72^{*}$ & $0.72^{\ddagger}$ & $0.81^{\ddagger}$ & $0.79^{\ddagger}$ \\
\hline & & Physical complaints & $0.50^{*}$ & $0.72^{\ddagger}$ & $0.71^{\ddagger}$ & $0.77^{\ddagger}$ \\
\hline & & Psychological issues & 0.29 & 0.40 & $0.62^{+}$ & $0.54^{*}$ \\
\hline & & Functional ability & $0.53^{*}$ & $0.54^{*}$ & $0.66^{\dagger}$ & $0.66^{\dagger}$ \\
\hline & & Emotional well-being & 0.19 & 0.42 & $0.57^{\dagger}$ & $0.50^{*}$ \\
\hline & & Extreme physical complaints & 0.38 & 0.34 & $0.63^{+}$ & $0.54^{*}$ \\
\hline & & Personal safety fears & 0.05 & 0.23 & $0.45^{*}$ & 0.34 \\
\hline Nishiyama et al., 2005 [24] & BDI & & $-0.55^{\ddagger}$ & $-0.77^{\S}$ & $-0.53^{\ddagger}$ & $-0.69^{\S}$ \\
\hline \multirow[t]{4}{*}{ Patel et al., 2012 [27] } & K-BILD & Total & $-0.67^{\dagger}$ & $-0.79^{\dagger}$ & $-0.87^{\dagger}$ & $-0.89^{\dagger}$ \\
\hline & & Psychological & $-0.60^{\dagger}$ & $-0.67^{\dagger}$ & $-0.80^{\dagger}$ & $-0.79^{\dagger}$ \\
\hline & & Breathlessness & $-0.59^{\dagger}$ & $-0.84^{\dagger}$ & $-0.80^{\dagger}$ & $-0.86^{\dagger}$ \\
\hline & & Chest & $-0.65^{\dagger}$ & $-0.64^{\dagger}$ & $-0.79^{\dagger}$ & $-0.78^{\dagger}$ \\
\hline Peng et al., 2008 [28] & Dyspnea score & & NS & $0.58^{\S}$ & $0.30^{+}$ & $0.38^{\ddagger}$ \\
\hline Swigris et al., 2012 [35] & UCSD-SOBQ & & & $0.80^{\S}$ & & \\
\hline \multirow[t]{3}{*}{ Yorke et al., 2010¹ [10] } & BDI & & $-0.39^{\S}$ & $-0.72^{\S}$ & $-0.61^{\S}$ & $-0.68^{\S}$ \\
\hline & SF-36 PCS & & $-0.52^{\S}$ & $-0.74^{\S}$ & $-0.63^{\S}$ & $-0.71^{\S}$ \\
\hline & Borg Dyspnea Index & & $0.35^{\S}$ & $0.45^{\S}$ & $0.40^{\S}$ & $0.45^{\S}$ \\
\hline Yorke et al., 2011 [39] & $D-12$ & & $0.57^{\ddagger}$ & $0.78^{\ddagger}$ & $0.75^{\ddagger}$ & $0.79^{\ddagger}$ \\
\hline Zimmermann et al., 2007 [40] & $\mathrm{BDI}$ & & $-0.62^{*}$ & $-0.75^{*}$ & $-0.63^{*}$ & $-0.72^{*}$ \\
\hline \multicolumn{7}{|l|}{ Longitudinal studies } \\
\hline Nishiyama et al., 2008 [25] & $\Delta \mathrm{BDI}$ & & & & & -0.29 \\
\hline Peng et al., 2008 [28] & $\Delta$ Dyspnea score & & NS & $0.59^{\dagger}$ & $0.56^{\dagger}$ & $0.45^{\dagger}$ \\
\hline
\end{tabular}

$\mathrm{BDI}=$ Baseline Dyspnea Index; CQLQ = Cough Quality of Life Questionnaire; D-12 = Dyspnea-12; K-BILD = King's Brief Interstitial Lung Disease questionnaire; SF-36 PCS = SF-36 Physical Component Summary; UCSD-SOBQ = University of California San Diego Shortness of Breath Questionnaire; $\Delta=$ change. ${ }^{*} p<0.05 ;{ }^{\dagger} p<0.01 ;{ }^{\ddagger} p<0.001 ;{ }^{\S} p<0.0001 ; N S=$ non-significant. ${ }^{1}$ Data reported refer to the original version of the SGRQ, not the SGRQ-I.

to -0.66 , and $\mathrm{p}<0.05$ for all but one). There were moderate to strong correlations between the SGRQ activity score and the majority of pertinent PFT results (e.g., FVC or $\mathrm{DL}_{\mathrm{CO}}$ ) or arterial blood gas analysis in all studies, while correlations between the SGRQ symptoms or impact domain scores and these variables were generally weak to moderate. Results for FVC, the lung function parameter regarded as the most statistically useful physiological indicator of IPF severity, and the one most frequently used as a primary endpoint in contemporary clinical trials, were weakly to moderately correlated with SGRQ total and domain scores $(r=-0.34$ to -0.45 for the SGRQ total and -0.13 to -0.31 for the SGRQ domains).

\section{HRCT}

In one study of patients with IPF, investigators assessed correlations between SGRQ scores and the extent of fibrotic abnormalities on HRCT (degree of ground-glass opacity [CT-alv], interstitial opacity [CT-fib], and both [total score]) (Table 5). Correlations were moderately strong between the SGRQ symptoms, impact and total scores and CT-alv or total scores $(r=0.34$ to 0.42$)$ and moderately strong between the SGRQ activity score and both the CT-fib and total scores $(r=0.37$ to 0.39$)$ [28].

\section{Known groups validity}

Although there are no well-established categories of disease severity in IPF, it may be hypothesized that patients receiving supplemental oxygen represent patients with more severe disease. In two studies, investigators found that SGRQ total scores were worse in patients using supplemental oxygen versus those not using supplemental oxygen [15,38]. In one study by Chang and colleagues, the magnitude of difference between patients using versus not using oxygen was 4.7 ( $p<0.05)$ [15]. 
Table 3 Correlation coefficients between SGRQ scores and the 6MWD as a measure of exercise capacity

\begin{tabular}{|c|c|c|c|c|c|}
\hline & Measure & $\begin{array}{l}\text { Correlation with } \\
\text { SGRQ symptoms } \\
\text { domain score }\end{array}$ & $\begin{array}{l}\text { Correlation with } \\
\text { SGRQ activity } \\
\text { domain score }\end{array}$ & $\begin{array}{l}\text { Correlation with } \\
\text { SGRQ impact } \\
\text { domain score }\end{array}$ & $\begin{array}{l}\text { Correlation } \\
\text { with SGRQ } \\
\text { total score }\end{array}$ \\
\hline \multicolumn{6}{|l|}{ Cross-sectional studies } \\
\hline Chang et al., 1999 [15] & $6 \mathrm{MWD}$ & & & & $-0.66^{\dagger}$ \\
\hline du Bois et al., 2011 [16] & $6 \mathrm{MWD}$ & & & & $-0.26^{\ddagger}$ \\
\hline Peng et al., 2008 [28] & $6 \mathrm{MWD}$ & $-0.32^{\dagger}$ & $-0.43^{\ddagger}$ & $-0.41^{\ddagger}$ & $-0.45^{\ddagger}$ \\
\hline Yorke et al., 2010¹ [10] & $6 \mathrm{MWD}$ & -0.14 & $-0.32^{\S}$ & $-0.24^{\dagger}$ & $-0.28^{\dagger}$ \\
\hline Yorke et al., 2011 [39] & $6 \mathrm{MWD}$ & $-0.32^{\dagger}$ & $-0.54^{\dagger}$ & $-0.47^{\dagger}$ & \\
\hline Zimmermann et al., 2007 [40] & $6 \mathrm{MWD}$ & -0.41 & $-0.72^{*}$ & $-0.63^{*}$ & $-0.72^{*}$ \\
\hline \multicolumn{6}{|l|}{ Longitudinal studies } \\
\hline du Bois et al., 2011 [16] & $\Delta 6 \mathrm{MWD}$ & & & & $-0.231^{\ddagger}$ \\
\hline Nishiyama et al., 2008 [25] & $\Delta 6 \mathrm{MWD}$ & & & & $-0.43^{*}$ \\
\hline Peng et al., 2008 [28] & $\Delta 6 \mathrm{MWD}$ & NS & -0.43 & -0.46 & $-0.41^{\dagger}$ \\
\hline
\end{tabular}

6MWD = Distance covered in 6-minute walk test; $\Delta=$ change.

${ }^{*} \mathrm{p}<0.05 ;{ }^{\dagger} \mathrm{p}<0.01 ;{ }^{\neq} \mathrm{p}<0.001 ;{ }^{\S} \mathrm{p}<0.0001 ; \mathrm{NS}=$ non-significant. ${ }^{1}$ Data reported refer to the original version of the SGRQ, not the SGRQ-I.

\section{Test-retest reliability (reproducibility)}

No studies were found that reported data on the testretest reliability of the SGRQ in patients with stable IPF.

\section{Minimal important difference}

A triangulation approach has been used to determine an MID estimate for SGRQ scores in patients with IPF [34]. Using both distribution- and anchor-based approaches (using FVC, DL $\mathrm{CO}$ and the TDI as anchors), the MID for the SGRQ symptoms, activity, impact and total scores was $8,5,7$ and 7 respectively.

\section{Responsiveness}

The responsiveness of the SGRQ domain and total scores has been assessed in one study [34]. Using data from a randomized placebo-controlled trial of bosentan, investigators assessed the ability of the SGRQ to discriminate among IPF patients who had experienced an improvement, decline, or no change in disease status over 6 months, as defined by three clinical anchors (change in FVC, DL $\mathrm{CO}_{\text {, }}$ transition dyspnea index [TDI]). With the exception of the SGRQ symptoms score when $\mathrm{DL}_{\mathrm{CO}}$ was the anchor, changes in SGRQ domain and total scores differed significantly between patients who had declined, remained stable, or improved. [34]. Change scores from the SGRQ total and its domains were reported for the $\mathrm{DL}_{\mathrm{CO}}$ and TDI response categories and ranged from +3 to $+13,+1$ to -5 , and 0 to -12 for patients that declined, remained stable, or improved, respectively. The impact domain discriminated best between all categories of change for all three anchors [34].

\section{SGRQ as an endpoint}

In sixteen trials, investigators used the SGRQ domain and/or total scores as outcome variables. In four trials, investigators evaluated the within-subject change in SGRQ total score from baseline to end of treatment $[22,23,32,37]$ (Table 6). In all four, improvements were observed in exercise endurance or FVC; among these, in three there was a significant decrease in SGRQ total score from baseline to end of treatment (8-24 weeks).

In the remaining 12 trials, investigators assessed whether the SGRQ domain and/or total scores differed between active and placebo groups (Table 7). In four of these $[13,17,18,25]$, statistically significant between-group differences for the primary endpoint coincided with statistically significant between-group differences in at least one SGRQ total or domain score (range of between-groups difference in SGRQ total score: -6.1 to -13.4). Six studies [17,20,26,29-31] reported a lack of statistically significant treatment effect in the primary endpoint or SGRQ scores (range of between-groups difference in SGRQ total score reported in three studies: -0.5 to -3.0 ; scores were not reported in three studies). In three studies [19,33,41], the primary endpoint was not met, but the SGRQ total or domain scores were significantly different between treatment groups (range of between-groups difference in SGRQ total score: -3.3 to -6.1$)$.

Four studies $[20,31,33,41]$ reported changes from baseline in SGRQ total score in the placebo group. Adjusting for different trial durations, the SGRQ total score in the placebo arms of these trials deteriorated (increased) by a median of +4.9 (range: 3.2 to 10.6 ) per 52 weeks.

\section{Floor and ceiling effects}

No studies were found in which investigators reported data on floor and ceiling effects for the SGRQ in patients with IPF. However, in most studies, the minimum and maximum achievable SGRQ total scores (0 and 100, respectively) were 
Table 4 Correlation coefficients between SGRQ scores, pulmonary function tests and arterial blood gas analysis

\begin{tabular}{|c|c|c|c|c|c|}
\hline & Lung function measure & $\begin{array}{l}\text { Correlation } \\
\text { with SGRQ } \\
\text { symptoms } \\
\text { domain score }\end{array}$ & $\begin{array}{l}\text { Correlation } \\
\text { with SGRQ } \\
\text { activity domain } \\
\text { score }\end{array}$ & $\begin{array}{l}\text { Correlation } \\
\text { with SGRQ } \\
\text { impact domain } \\
\text { score }\end{array}$ & $\begin{array}{l}\text { Correlation } \\
\text { with SGRQ } \\
\text { total score }\end{array}$ \\
\hline \multirow[t]{4}{*}{ Chang et al., 1999 [15] } & DLco\% predicted & & & & $-0.55^{\dagger}$ \\
\hline & $\mathrm{FEV}_{1} \%$ predicted & & & & $-0.46^{\dagger}$ \\
\hline & FVC\% predicted & & & & $-0.45^{\dagger}$ \\
\hline & TLC\% predicted & & & & $-0.36^{\dagger}$ \\
\hline \multirow[t]{5}{*}{ Nishiyama et al., 2005 [24] } & $\mathrm{PaO}_{2}$ & -0.21 & $-0.48^{\dagger}$ & -0.29 & $-0.37^{*}$ \\
\hline & $\mathrm{SpO}_{2}$ & $-0.38^{*}$ & $-0.48^{\dagger}$ & -0.22 & $-0.37^{*}$ \\
\hline & TLC & $-0.48^{\dagger}$ & $-0.38^{*}$ & -0.21 & $-0.36^{*}$ \\
\hline & $T L_{C O}$ & $-0.32^{*}$ & $-0.45^{\dagger}$ & -0.27 & $-0.39^{*}$ \\
\hline & VC & $-0.35^{*}$ & $-0.36^{*}$ & -0.15 & -0.30 \\
\hline \multirow[t]{5}{*}{ Peng et al., 2008 [28] } & $\mathrm{DL}_{\mathrm{CO}} \%$ predicted & $-0.46^{\S}$ & $-0.46^{\S}$ & $-0.34^{\dagger}$ & $-0.44^{\ddagger}$ \\
\hline & $\mathrm{FEV}_{1} \%$ predicted & NS & $-0.53^{\S}$ & $-0.34^{\dagger}$ & $-0.42^{\S}$ \\
\hline & $\mathrm{PaO}_{2}$ & NS & $-0.54^{\ddagger}$ & NS & $-0.32^{\dagger}$ \\
\hline & TLC\% predicted & $-0.50^{\S}$ & $-0.61^{\S}$ & $-0.52^{\S}$ & $-0.62^{\S}$ \\
\hline & VC\% predicted & NS & $-0.59^{\S}$ & $-0.35^{\dagger}$ & $-0.47^{\S}$ \\
\hline \multirow[t]{4}{*}{ Tzanakis et al., 2005 [36] } & $\mathrm{FEV}_{1} \%$ predicted & & & & $-0.50^{\dagger}$ \\
\hline & $\mathrm{PaO}_{2}$ (at rest) & & & & $-0.51^{\dagger}$ \\
\hline & $\mathrm{PaO}_{2}$ (at exertion) & & & & $-0.60^{\dagger}$ \\
\hline & TLC\% predicted & & & & $-0.55^{\dagger}$ \\
\hline \multirow[t]{2}{*}{ Yorke et al., 2010' [10] } & FVC\% predicted & $-0.27^{\dagger}$ & $-0.31^{\S}$ & $-0.30^{\S}$ & $-0.34^{\S}$ \\
\hline & $\mathrm{TL}_{\mathrm{CO}} \%$ predicted & $-0.23^{+}$ & $-0.34^{\S}$ & $-0.38^{\S}$ & $-0.38^{\S}$ \\
\hline \multirow[t]{2}{*}{ Yorke et al., 2011 [39] } & $\mathrm{DL}_{\mathrm{CO}} \%$ & -0.16 & $-0.37^{\dagger}$ & $-0.28^{*}$ & \\
\hline & FVC\% & -0.13 & -0.16 & $-0.24^{*}$ & \\
\hline \multirow[t]{4}{*}{ Zimmermann et al., 2007 [40] } & $\mathrm{DL}_{\mathrm{CO}} \%$ predicted & -0.41 & -0.32 & -0.39 & $-0.47^{*}$ \\
\hline & $\mathrm{FEV}_{1} \%$ predicted & -0.08 & $-0.57^{*}$ & $-0.52^{*}$ & $-0.57^{*}$ \\
\hline & TLC\% predicted & -0.37 & $-0.65^{*}$ & $-0.58^{*}$ & $-0.66^{*}$ \\
\hline & VC\% predicted & -0.14 & $-0.54^{*}$ & $-0.61^{*}$ & $-0.56^{*}$ \\
\hline
\end{tabular}

$\mathrm{DL}_{\mathrm{CO}}=$ diffusion capacity of the lung for carbon monoxide; $\mathrm{FEV}_{1}=$ forced expiratory volume in 1 second; FVC = forced vital capacity; PaO $\mathrm{F}_{2}=$ partial pressure of oxygen dissolved in arterial blood; $\mathrm{TLC}=$ total lung capacity, $\mathrm{TL}_{\mathrm{CO}}=$ transfer factor of the lung for carbon monoxide; $\mathrm{VC}=$ vital capacity.

${ }^{*} \mathrm{p}<0.05 ;{ }^{\dagger} \mathrm{p}<0.01 ;{ }^{\ddagger} \mathrm{p}<0.001 ;{ }^{\S} \mathrm{p}<0.0001 ; \mathrm{NS}=$ non-significant. ${ }^{1}$ Data reported refer to the original version of the SGRQ, not the SGRQ-I.

outside an interval spanning twice the standard deviation around the reported means (Table 1). For the two studies in which investigators reported ranges for baseline SGRQ total scores, ranges did not include minimum or maximum possible values $[24,38]$, thus confirming the absence of floor or ceiling effects in these studies.

\section{Conclusions}

Measurement standards and psychometric criteria have been proposed to assist with choosing an appropriate instrument to evaluate HRQL in patients with IPF $[6,43]$. As with any patient-reported outcome measure used in the study of any condition, an instrument must have face

Table 5 Correlation coefficients between SGRQ scores and extent of fibrosis on HRCT

\begin{tabular}{llllll}
\hline Study & HRCT measure & $\begin{array}{l}\text { Correlation with } \\
\text { SGRQ symptoms } \\
\text { domain score }\end{array}$ & $\begin{array}{l}\text { Correlation with } \\
\text { SGRQ activity } \\
\text { domain score }\end{array}$ & $\begin{array}{l}\text { Correlation with } \\
\text { SGRQ impact } \\
\text { domain score }\end{array}$ & $\begin{array}{l}\text { Correlation } \\
\text { with SGRQ } \\
\text { total score }\end{array}$ \\
\hline Peng et al., 2008 [28] & CT-alv & $0.41^{\dagger}$ & $\mathrm{NS}$ & $0.34^{*}$ & $\mathrm{~N}$ \\
& CT-fib & $\mathrm{NS}$ & $0.37^{*}$ & $\mathrm{NS}$ & $\mathrm{NS}$ \\
& CT-tot & $0.36^{*}$ & $0.39^{\dagger}$ & $0.35^{*}$ & $0.42^{\dagger}$ \\
\hline
\end{tabular}

CT-alv = ground glass opacity; $\mathrm{CT}$-fib = interstitial opacity; $\mathrm{CT}$-tot $=$ total.

${ }^{*} \mathrm{p}<0.01 ;{ }^{\dagger} \mathrm{p}<0.001 ; \mathrm{NS}=$ non-significant. 
Table 6 Changes in SGRQ scores in within-subject clinical trials

\begin{tabular}{|c|c|c|c|c|c|c|c|c|}
\hline \multirow[t]{2}{*}{ Study } & \multirow{2}{*}{$\begin{array}{l}\text { Treatment under } \\
\text { investigation }\end{array}$} & \multirow{2}{*}{$\begin{array}{l}\text { Treatment } \\
\text { duration }\end{array}$} & \multicolumn{2}{|c|}{ Sample size } & \multicolumn{2}{|c|}{ SGRQ total score ${ }^{1}$} & \multirow{2}{*}{$\begin{array}{l}\text { Effect } \\
\text { size }\end{array}$} & \multirow[t]{2}{*}{ p-value ${ }^{2}$} \\
\hline & & & Total & IPF & Baseline & Post-treatment & & \\
\hline Mishra et al., 2011 [22] & Oral doxycycline & 24 weeks & 6 & 6 & $50.90(8.38)$ & $18.40(6.39)$ & 3.88 & $<0.001$ \\
\hline Naji et al., 2006 [23] & Pulmonary rehabilitation & 8 weeks & 26 & 19 & $48.3[21.5,82]$ & $39.5[17.4,69.4]$ & 0.41 & $<0.10$ \\
\hline Rammaert et al., 2009 [32] & Pulmonary rehabilitation & 8 weeks & 13 & 13 & - & - & - & NS \\
\hline Tzouvelekis et al., 2013 [37] & $\begin{array}{l}\text { Endobronchial infusion of } \\
\text { adipose-derived stromal cells }\end{array}$ & 6 months & 14 & 14 & $35.1(6.8)$ & $27.8(5.6)$ & 1.07 & $<0.05$ \\
\hline
\end{tabular}

${ }^{1}$ Mean (SD) or median [range] are reported based on availability.

${ }^{2} \mathrm{p}$-value for test of statistical significance between SGRQ score at baseline and post-treatment.

validity, internal consistency, test-retest reliability, longitudinal validity, and minimal floor and ceiling effects in the target patient population.

The constellation of findings from studies identified in our search revealed that in patients with IPF, the internal consistency of the SGRQ activity and impact domains and the SGRQ total score was excellent, and the internal consistency of the symptoms domain was moderate, and in most studies, fell below the acceptable threshold of 0.7. The lower internal consistency of the symptoms domain is likely because it asks about a range of respiratory symptoms (cough, sputum, shortness of breath, wheezing and attacks of chest trouble), the majority of which apply to few patients with IPF whose major symptoms are shortness of breath and cough. In response data, offtarget items create a weaker level of inter-relatedness among items in this domain, and thus lower internal consistency. This also contributes to the lower convergent validity of this domain, as the off-target items weaken the associations between its scores and clinical measures of IPF severity (e.g., patients may endorse wheezing or attacks of chest trouble, but these symptoms are unlikely related to a person's FVC). These offtarget (for IPF) items in the symptoms domain detract from the SGRQ's face validity and would likely have been removed or modified in a tool specifically designed for use with patients with IPF. Overall, the symptoms domain may be well-suited for patients with COPD, but is not tailored to precisely assess symptoms in patients with IPF. The non-informative noise in the symptoms domain might also contribute to a less than optimal performance of the SGRQ total score. Overall, however, despite its weak face validity in IPF, the symptoms domain performs reasonably well in this population, and its potential to detract from the performance of the SGRQ total score is tempered because it contributes least to the SGRQ total score.

Convergent validity analyses seek to determine whether two measures, hypothesized to measure the same construct, do in fact correlate, and moderate, statistically significant correlations in the expected direction support convergent validity. Very strong or 'perfect' correlations, suggest redundancy in measurement, so moderate correlations between a patient-reported outcome measure and another clinical variable support convergent validity of the patient-reported outcome measure while confirming that it contributes unique information not captured by the other clinical variable [5]. The SGRQ has been used as a secondary endpoint in several clinical trials conducted in patients with IPF. Among the select few in which the intervention outperformed placebo, SGRQ results were as one would anticipate, i.e., SGRQ scores improved in the group that benefited from the intervention. Although not a formal assessment of responsiveness, consistency between the changes in SGRQ scores and the changes in other endpoints supports responsiveness.

In sum, the limitations of the SGRQ in IPF should be noted, as it was not originally developed for use in patients with IPF. In particular, this applies to possible over-interpretation of results of individual domains. However, the cross-sectional correlations between SGRQ domain and total scores and other measures of patientreported health status, exercise capacity or lung function, along with the ability of the SGRQ to distinguish patients who experience a change in clinical status or remain stable over time, support the SGRQ as a useful patient-reported outcome measure in IPF.

Limitations to our research include the following: we could only identify one study in which MID estimates for the SGRQ scores in IPF were determined [44]. This study used a triangulation approach and concluded an MID that was higher than that reported for COPD [45], but more research with additional datasets is needed to evaluate these estimates. In the meantime, the use of responder rates of patients experiencing a minimum change from baseline in SGRQ scores - or perhaps more informative, cumulative distribution plots - may be a useful assessment, as research suggests that it may be less dependent on the exact cutoff, i.e. the precise value of the MID [46].

No articles were identified that evaluated the testretest reliability of the SGRQ in patients with stable IPF. Likewise, we could not locate a study in which floor and ceiling effects of SGRQ scores were reported, although an analysis of the reported baseline mean SGRQ total 
Table 7 Changes in SGRQ scores in randomized controlled trials

\begin{tabular}{|c|c|c|c|c|c|c|c|}
\hline Study & $\begin{array}{l}\text { Treatment } \\
\text { duration }\end{array}$ & $\begin{array}{l}\text { Sample } \\
\text { size, IPF }\end{array}$ & Randomized groups & $\begin{array}{l}\text { Change from } \\
\text { baseline in } \\
\text { SGRQ symptoms } \\
\text { domain score }\end{array}$ & $\begin{array}{l}\text { Change from } \\
\text { baseline in } \\
\text { SGRQ activity } \\
\text { domain score }\end{array}$ & $\begin{array}{l}\text { Change from } \\
\text { baseline in } \\
\text { SGRQ impact } \\
\text { domain score }\end{array}$ & $\begin{array}{l}\text { Change from } \\
\text { baseline in } \\
\text { SGRQ total } \\
\text { score }\end{array}$ \\
\hline \multirow{3}{*}{$\begin{array}{l}\text { Antoniou et al., } \\
2006 \text { [13] }\end{array}$} & \multirow[t]{3}{*}{12 months } & \multirow[t]{3}{*}{50} & Interferon gamma b & $-13.2[21.4,5.0]$ & $-4.8[-12.7,3.0]$ & $-1.9[-9.2,5.4]$ & $-4.7[-11.4,2.0]$ \\
\hline & & & Colchicine & $7.5[-4.5,19.5]$ & $4.7[-12.1,22.0]$ & $4.1[-6.4,14.6]$ & $4.8[-5.9,15.5]$ \\
\hline & & & p-value ${ }^{2}$ & 0.01 & NS & NS & NS \\
\hline \multirow{8}{*}{$\begin{array}{l}\text { Han et al., } \\
2013 \text { [17] }\end{array}$} & \multirow[t]{8}{*}{12 weeks } & \multirow[t]{4}{*}{22} & Sildenafil (with RVSD) & - & - & - & - \\
\hline & & & Placebo (with RVSD) & - & - & - & - \\
\hline & & & Difference ${ }^{1}$ & $-28.0[-41.7,-14.4]$ & $-5.6[-16.1,5.0]$ & $-14.0[-25.6,-2.4]$ & $-13.4[-22.7,-4.2]$ \\
\hline & & & p-value ${ }^{2}$ & $<0.0001$ & NS & 0.02 & 0.005 \\
\hline & & \multirow[t]{4}{*}{97} & Sildenafil (without RVSD) & - & - & - & - \\
\hline & & & Placebo, (without RVSD) & - & - & - & - \\
\hline & & & Difference $^{1}$ & $-3.8[-10.7,3.0]$ & $-4.1[-9.2,1.1]$ & $-1.8[-7.5,3.9]$ & $-3.0[-7.6,1.7]$ \\
\hline & & & p-value ${ }^{2}$ & NS & NS & NS & NS \\
\hline \multirow{4}{*}{$\begin{array}{l}\text { Horton et al., } \\
2012 \text { [18] }\end{array}$} & \multirow[t]{4}{*}{12 weeks } & \multirow[t]{4}{*}{23} & Thalidomide & - & - & - & - \\
\hline & & & Placebo & - & - & - & - \\
\hline & & & Difference $^{1}$ & $-12.1[22.2,2.0]$ & $-3.3[-9.8,3.2]$ & $-13.1[-19.7,-6.6]$ & $-11.7[-18.6,-4.8]$ \\
\hline & & & $p$-value ${ }^{2}$ & 0.018 & NS & $<0.001$ & 0.001 \\
\hline \multirow{4}{*}{$\begin{array}{l}\text { King, Jr. et al., } \\
2008 \text { [19] }\end{array}$} & \multirow[t]{4}{*}{6 months $^{4}$} & \multirow[t]{4}{*}{158} & Bosentan & - & - & - & - \\
\hline & & & Placebo & - & - & - & - \\
\hline & & & Difference $^{1}$ & - & - & - & $-3.3(2.6)$ \\
\hline & & & $p$-value ${ }^{2}$ & - & - & - & 0.034 \\
\hline \multirow{3}{*}{$\begin{array}{l}\text { King, Jr. et al., } \\
2009 \text { [20] }\end{array}$} & \multirow[t]{3}{*}{77 weeks } & \multirow[t]{3}{*}{826} & Interferon gamma b & - & - & - & $5.7(13.5)$ \\
\hline & & & Placebo & - & - & - & $6.2(14.3)$ \\
\hline & & & $p$-value ${ }^{2}$ & - & - & - & NS \\
\hline \multirow{4}{*}{$\begin{array}{l}\text { Nishiyama et al., } \\
2008 \text { [25] }\end{array}$} & \multirow[t]{4}{*}{10 weeks } & \multirow[t]{4}{*}{28} & Pulmonary rehabilitation & - & - & - & - \\
\hline & & & No pulmonary rehabilitation & - & - & - & - \\
\hline & & & Difference $^{1}$ & $-5.7[-18.7,7.2]$ & $-5.8[-14.7,3.1]$ & $-6.2[-12.8,0.3]$ & $-6.1[-11.7,0.5]$ \\
\hline & & & $p$-value ${ }^{2}$ & NS & NS & NS & $<.05$ \\
\hline \multirow{3}{*}{$\begin{array}{l}\text { Noth et al., } \\
2012 \text { [26] }\end{array}$} & \multirow[t]{3}{*}{28 weeks } & \multirow[t]{3}{*}{145} & Warfarin & - & - & - & - \\
\hline & & & Placebo & - & - & - & - \\
\hline & & & $p$-value ${ }^{2}$ & - & - & - & NS \\
\hline \multirow{3}{*}{$\begin{array}{l}\text { Raghu et al., } \\
2004 \text { [29] }\end{array}$} & \multirow[t]{3}{*}{48 weeks } & \multirow[t]{3}{*}{330} & Interferon gamma $1 \mathrm{~b}$ & - & - & - & - \\
\hline & & & Placebo & - & - & - & - \\
\hline & & & $p$-value ${ }^{2}$ & - & - & - & NS \\
\hline Raghu et al., & 48 weeks & 88 & Etanercept & - & - & - & - \\
\hline & & & Placebo & - & - & - & - \\
\hline & & & Difference $^{1}$ & - & - & - & - \\
\hline & & & $p$-value ${ }^{2}$ & NS & NS & NS & NS \\
\hline Raghu et al., & 48 weeks & 492 & Ambrisentan & - & - & - & 4.7 \\
\hline & & & Placebo & - & - & - & 3.0 \\
\hline & & & $p$-value ${ }^{2}$ & - & - & - & NS \\
\hline
\end{tabular}


Table 7 Changes in SGRQ scores in randomized controlled trials (Continued)

\begin{tabular}{|c|c|c|c|c|c|c|c|}
\hline \multirow{6}{*}{$\begin{array}{l}\text { Richeldi et al., } \\
2011 \text { [33] }\end{array}$} & \multirow[t]{6}{*}{12 months } & \multirow[t]{6}{*}{431} & Nintedanib 50 mg qd & $3.39(2.51)$ & $7.39(1.96)$ & $3.71(2.04)$ & $4.67(1.78)$ \\
\hline & & & Nintedanib 50 mg bid & $2.11(2.34)$ & $3.54(1.82)$ & $1.73(1.90)$ & $2.18(1.65)$ \\
\hline & & & Nintedanib 100 mg bid & $2.33(2.35)$ & $3.00(1.83)$ & $0.79(1.91)$ & $1.48(1.66)$ \\
\hline & & & Nintedanib 150 mg bid & $-3.14(2.40)$ & $0.32(1.89)$ & $-0.14(1.97)$ & $-0.66(1.71)$ \\
\hline & & & Placebo & $6.45(2.45)$ & $7.48(1.91)$ & $4.21(1.99)$ & $5.46(1.73)$ \\
\hline & & & p-value ${ }^{3}$ & $<0.005$ & $<0.005$ & - & $<0.01$ \\
\hline \multirow{4}{*}{$\begin{array}{l}\text { Zisman et al., } \\
2010 \text { [41] }\end{array}$} & \multirow[t]{4}{*}{12 weeks } & \multirow[t]{4}{*}{180} & Sildenafil & $-3.58[-7.02,-0.13]$ & $-1.15[-3.68,1.38]$ & $-0.88[-3.78,2.02]$ & $-1.64[-3.91,0.64]$ \\
\hline & & & Placebo & $2.15[-1.30,5.61]$ & $2.49[0.00,4.99]$ & $2.82[-0.03,5.67]$ & $2.45[0.17,4.72]$ \\
\hline & & & Difference $^{1}$ & $-5.73[-10.61,-0.85]$ & $-3.64[-7.20,-0.09]$ & $-3.70[-7.76,0.37]$ & $-4.08[-7.30,-0.86]$ \\
\hline & & & p-value ${ }^{2}$ & 0.02 & .04 & NS & .01 \\
\hline
\end{tabular}

RVSD = right ventricular systolic dysfunction.

${ }^{1}$ Difference in change from baseline between treatment groups, mean $[95 \% \mathrm{Cl}]$.

${ }^{2}$ Test of statistical significance for the difference in mean change from baseline between groups.

${ }^{3}$ Test of statistical significance for the difference in mean change from baseline between the nintedanib $150 \mathrm{mg}$ bid and placebo groups.

${ }^{4}$ Treatment continued for $\geq 12$ months (data not available).

scores and their standard deviations suggested that there was no evidence for either. Furthermore, we did not assess the content validity of the SGRQ in patients with IPF, nor did we include analyses of articles published in languages other than English. Content validity and cultural adaption are important factors to consider for any patient-reported outcome measure, but these topics were beyond the scope of this evaluation of the SGRQ's psychometric properties. Therefore, it is evident that more research on the SGRQ is needed in this patient population.

The utility of a patient-reported outcome measure may be assessed only after a wealth of data becomes available. The assessment involves examining how the measure performs in the target population under several circumstances. The cache of available data has greatly advanced our understanding of HRQL in general, and the performance of the SGRQ in patients with IPF. For example, whilst the mean baseline SGRQ total score reported in IPF (around 45; interquartile range: 42-50) is similar to that reported in COPD trials $[47,48]$, an analysis of the reported changes from baseline in the SGRQ total score in the placebo arms suggests that untreated patients with IPF deteriorate by +4.9 points over a period of 52 weeks. This contrasts with the experience in COPD, where patients on placebo show an improvement of 2-3 points per year [46], and reflects the progressive decline in health status seen in patients with IPF.

Finally, a major factor in this assessment revolves around how confidently response data from the measure can be used to make inferences about patients in the target population. For example, what can be said about a patient with IPF whose SGRQ score is 50? How does day-to-day functioning, or how a patient feels, change for an IPF patient whose SGRQ score increases by 10 over 6 months? Being able to answer these, and similar, questions confidently and accurately will further and more strongly support the validity of the SGRQ as an instrument capable of assessing domains of HRQL in this population. Until then, the balance of the data suggests that the SGRQ may be a suitable secondary endpoint for measuring HRQL in therapeutic trials of IPF.

\section{Additional file}

Additional file 1: PubMed search strategy.

\section{Abbreviations}

6MWD: Distance covered in 6-minute walk test; BDI: Baseline Dyspnea Index; Bid: Twice daily; C: Comparator group; COPD: Chronic obstructive pulmonary disease; CQLQ: Cough Quality of Life Questionnaire; CT-alv: Degree of groundglass opacity; CT-fib: Interstitial opacity; CT-tot: Total; D-12: Dyspnea-12; $\mathrm{DL}_{\mathrm{co}}$ : Diffusing capacity for carbon monoxide; $\mathrm{FEV}_{1}$ : Forced expiratory volume in 1 second; FVC: Forced vital capacity; HRCT: High-resolution computed tomography; HRQL: Health-related quality of life; IPF: Idiopathic pulmonary fibrosis; K-BILD: King's Brief Interstitial Lung Disease questionnaire; MID: Minimal important difference; $\mathrm{PaO}_{2}$ : Partial pressure of oxygen dissolved in arterial blood; PFT: Pulmonary function tests; Qd: Once daily; RCT: Randomized controlled trial; RVSD: Right ventricular systolic dysfunction; SF-36 PCS: Short Form-36 Physical Component Summary score; SGRQ: St George's Respiratory Questionnaire; T: Treatment group; TDI: Transition dyspnea index; TLC: Total lung capacity; $\mathrm{TL}_{\mathrm{Co}}$ : Transfer factor of the lung for carbon monoxide; UCSD-SOBQ: University of California San Diego Shortness of Breath Questionnaire; VC: Vital capacity.

\section{Competing interests}

JJS has served as a paid consultant for Boehringer Ingelheim and InterMune. KKB has served as a paid consultant for Boehringer Ingelheim. DE and CSC are full-time employees of Boehringer Ingelheim. If accepted for publication in Health and Quality of Life Outcomes, the article processing charge for this article would be covered by Boehringer Ingelheim.

\section{Authors' contributions}

JJS interpreted the data and prepared the manuscript. DE conducted the literature search, extracted and interpreted the data, and prepared the manuscript. CSC interpreted the data and prepared the manuscript. KKB interpreted the data and prepared the manuscript. All authors approved the final version of the manuscript. 


\section{Acknowledgments}

The authors are grateful to Nancy Kline Leidy and Hilary Wilson of Evidera for their expert advice. Editorial assistance, supported financially by Boehringer Ingelheim, was provided by Wendy Morris and Julie Fleming of FleishmanHillard Group, Ltd, London, UK, during the preparation of this article. The authors were fully responsible for all content and editorial decisions, were involved at all stages of manuscript development and have approved the final version.

\section{Author details}

${ }^{1}$ National Jewish Health, Denver, CO, USA. ${ }^{2}$ Boehringer Ingelheim GmbH, Ingelheim am Rhein, Germany. ${ }^{3}$ Boehringer Ingelheim Pharmaceuticals, Inc., Ridgefield, CT, USA.

Received: 25 April 2014 Accepted: 5 August 2014 Published: 20 August 2014

\section{References}

1. Raghu G, Collard HR, Egan JJ, Martinez FJ, Behr J, Brown KK, Colby TV, Cordier JF, Flaherty KR, Lasky JA, Lynch DA, Ryu JH, Swigris JJ, Wells AU, Ancochea J, Bouros D, Carvalho C, Costabel U, Ebina M, Hansell DM, Johkoh T, Kim DS, King TE Jr, Kondoh Y, Myers J, Müller NL, Nicholson AG, Richeldi L, Selman M, Dudden RF, et al: An official ATS/ERS/JRS/ALAT statement: idiopathic pulmonary fibrosis: evidence-based guidelines for diagnosis and management. Am J Respir Crit Care Med 2011, 183:788-824.

2. Nalysnyk L, Cid-Ruzafa J, Rotella P, Esser D: Incidence and prevalence of idiopathic pulmonary fibrosis: review of the literature. Eur Respir Rev 2012, 21:355-361.

3. Ley B, Collard HR, King TE Jr: Clinical course and prediction of survival in idiopathic pulmonary fibrosis. Am J Respir Crit Care Med 2011, 183:431-440.

4. Swigris JJ, Stewart AL, Gould MK, Wilson SR: Patients' perspectives on how idiopathic pulmonary fibrosis affects the quality of their lives. Health Qual Life Outcomes 2005, 3:61.

5. Swigris JJ, Kuschner WG, Jacobs SS, Wilson SR, Gould MK: Health-related quality of life in patients with idiopathic pulmonary fibrosis: a systematic review. Thorax 2005, 60:588-594.

6. Belkin A, Swigris JJ: Health-related quality of life in idiopathic pulmonary fibrosis: where are we now? Curr Opin Pulm Med 2013, 19:474-479.

7. Swigris JJ, Gould MK, Wilson SR: Health-related quality of life among patients with idiopathic pulmonary fibrosis. Chest 2005, 127:284-294.

8. Swigris JJ, Fairclough D: Patient-reported outcomes in idiopathic pulmonary fibrosis research. Chest 2012, 142:291-297.

9. Jones PW, Quirk FH, Baveystock CM: The St George's Respiratory Questionnaire. Respir Med 1991, 85:25-31.

10. Yorke J, Jones PW, Swigris JJ: Development and validity testing of an IPF-specific version of the St George's Respiratory Questionnaire. Thorax 2010, 65:921-926.

11. Hays R, Revicki DA: Reliability and validity (including responsiveness). In Assessing quality of life in clinical trials. 2nd edition. Edited by Fayers P, Hays R. New York: Oxford University Press; 2005:25-39.

12. Hinkle D, Wiersma W, Jurs S: Applied statistics for the behavioral sciences. Boston: Houghton-Mifflin; 1988

13. Antoniou KM, Nicholson AG, Dimadi M, Malagari K, Latsi P, Rapti A, Tzanakis N, Trigidou R, Polychronopoulos V, Bouros D: Long-term clinical effects of interferon gamma-1b and colchicine in idiopathic pulmonary fibrosis. Eur Respir J 2006, 28:496-504.

14. Berry CE, Drummond MB, Han MK, Li D, Fuller C, Limper AH, Martinez FJ, Schwarz MI, Sciurba FC, Wise RA: Relationship between lung function impairment and health-related quality of life in COPD and interstitial lung disease. Chest 2012, 142:704-711.

15. Chang JA, Curtis JR, Patrick DL, Raghu G: Assessment of health-related quality of life in patients with interstitial lung disease. Chest 1999, 116:1175-1182.

16. Du Bois RM, Weycker D, Albera C, Bradford WZ, Costabel U, Kartashov A, Lancaster L, Noble PW, Sahn SA, Szwarcberg J, Thomeer M, Valeyre D, King TE Jr: Six-minute-walk test in idiopathic pulmonary fibrosis: test validation and minimal clinically important difference. Am J Respir Crit Care Med 2011, 183:1231-1237.

17. Han MK, Bach DS, Hagan PG, Yow E, Flaherty KR, Toews GB, Anstrom KJ, Martinez FJ, IPFnet Investigators: Sildenafil preserves exercise capacity in patients with idiopathic pulmonary fibrosis and right-sided ventricular dysfunction. Chest 2013, 143:1699-1708.

18. Horton MR, Santopietro V, Mathew L, Horton KM, Polito AJ, Liu MC, Danoff SK, Lechtzin N: Thalidomide for the treatment of cough in idiopathic pulmonary fibrosis: a randomized trial. Ann Intern Med 2012, 157:398-406.

19. King TE Jr, Behr J, Brown KK, Du Bois RM, Lancaster L, De Andrade JA, Stähler G, Leconte I, Roux S, Raghu G: BUILD-1: a randomized placebocontrolled trial of bosentan in idiopathic pulmonary fibrosis. Am J Respir Crit Care Med 2008, 177:75-81.

20. King TE Jr, Albera C, Bradford WZ, Costabel U, Hormel P, Lancaster L, Noble PW, Sahn SA, Szwarcberg J, Thomeer M, Valeyre D, Du Bois RM, INSPIRE Study Group: Effect of interferon gamma-1b on survival in patients with idiopathic pulmonary fibrosis (INSPIRE): a multicentre, randomised, placebo-controlled trial. Lancet 2009, 374:222-228.

21. Lechtzin N, Hilliard ME, Horton MR: Validation of the Cough Quality-of-Life Questionnaire in patients with idiopathic pulmonary fibrosis. Chest 2013, 143:1745-1749.

22. Mishra A, Bhattacharya P, Paul S, Paul R, Swarnakar S: An alternative therapy for idiopathic pulmonary fibrosis by doxycycline through matrix metalloproteinase inhibition. Lung India 2011, 28:174-179.

23. Naji NA, Connor MC, Donnelly SC, McDonnell TJ: Effectiveness of pulmonary rehabilitation in restrictive lung disease. J Cardiopulm Rehabil 2006, 26:237-243.

24. Nishiyama O, Taniguchi $H$, Kondoh Y, Kimura T, Ogawa T, Watanabe F, Nishimura K: Health-related quality of life in patients with idiopathic pulmonary fibrosis. What is the main contributing factor? Respir Med 2005, 99:408-414.

25. Nishiyama O, Kondoh Y, Kimura T, Kato K, Kataoka K, Ogawa T, Watanabe F, Arizono S, Nishimura K, Taniguchi H: Effects of pulmonary rehabilitation in patients with idiopathic pulmonary fibrosis. Respirology 2008, 13:394-399.

26. Noth I, Anstrom KJ, Calvert SB, De Andrade J, Flaherty KR, Glazer C, Kaner RJ, Olman MA, Idiopathic Pulmonary Fibrosis Clinical Research Network (IPFnet): A placebo-controlled randomized trial of warfarin in idiopathic pulmonary fibrosis. Am J Respir Crit Care Med 2012, 186:88-95.

27. Patel AS, Siegert RJ, Brignall K, Gordon P, Steer S, Desai SR, Maher TM, Renzoni EA, Wells AU, Higginson IJ, Birring SS: The development and validation of the King's Brief Interstitial Lung Disease (K-BILD) health status questionnaire. Thorax 2012, 67:804-810.

28. Peng S, Li Z, Kang J, Hou X: Cross-sectional and longitudinal construct validity of the Saint George's Respiratory Questionnaire in patients with IPF. Respirology 2008, 13:871-879.

29. Raghu G, Brown KK, Bradford WZ, Starko K, Noble PW, Schwartz DA, King TE Jr, Idiopathic Pulmonary Fibrosis Study Group: A placebo-controlled trial of interferon gamma-1b in patients with idiopathic pulmonary fibrosis. N Engl J Med 2004, 350:125-133.

30. Raghu G, Brown KK, Costabel U, Cottin V, Du Bois RM, Lasky JA, Thomeer M, Utz JP, Khandker RK, McDermott L, Fatenejad S: Treatment of idiopathic pulmonary fibrosis with etanercept: an exploratory, placebo-controlled trial. Am J Respir Crit Care Med 2008, 178:948-955.

31. Raghu G, Behr J, Brown KK, Egan JJ, Kawut SM, Flaherty KR, Martinez FJ, Nathan SD, Wells AU, Collard HR, Costabel U, Richeldi L, De Andrade J, Khalil N, Morrison LD, Lederer DJ, Shao L, Li X, Pedersen PS, Montgomery AB, Chien JW, O'Riordan TG, ARTEMIS-IPF Investigators: Treatment of idiopathic pulmonary fibrosis with ambrisentan: a parallel, randomized trial. Ann Intern Med 2013, 158:641-649.

32. Rammaert B, Leroy S, Cavestri B, Wallaert B, Grosbois JM: Home-based pulmonary rehabilitation in idiopathic pulmonary fibrosis. Rev Mal Respir 2009, 26:275-282.

33. Richeldi L, Costabel U, Selman M, Kim DS, Hansell DM, Nicholson AG, Brown KK, Flaherty KR, Noble PW, Raghu G, Brun M, Gupta A, Juhel N, Klüglich M, Du Bois RM: Efficacy of a tyrosine kinase inhibitor in idiopathic pulmonary fibrosis. N Engl J Med 2011, 365:1079-1087.

34. Swigris JJ, Brown KK, Behr J, Du Bois RM, King TE, Raghu G, Wamboldt FS: The SF-36 and SGRQ: validity and first look at minimum important differences in IPF. Respir Med 2010, 104:296-304.

35. Swigris JJ, Han M, Vij R, Noth I, Eisenstein EL, Anstrom KJ, Brown KK, Fairclough D: The UCSD shortness of breath questionnaire has longitudinal construct validity in idiopathic pulmonary fibrosis. Respir Med 2012, 106:1447-1455.

36. Tzanakis N, Samiou M, Lambiri I, Antoniou K, Siafakas N, Bouros D: Evaluation of health-related quality-of-life and dyspnea scales in patients 
with idiopathic pulmonary fibrosis. Correlation with pulmonary function tests. Eur J Intern Med 2005, 16:105-112.

37. Tzouvelekis A, Paspaliaris V, Koliakos G, Ntolios P, Bouros E, Oikonomou A, Zissimopoulos A, Boussios N, Dardzinski B, Gritzalis D, Antoniadis A, Froudarakis M, Kolios G, Bouros D: A prospective, non-randomized, no placebo-controlled, phase $\mathrm{lb}$ clinical trial to study the safety of the adipose derived stromal cells-stromal vascular fraction in idiopathic pulmonary fibrosis. J Trans/ Med 2013, 11:171.

38. Verma G, Marras T, Chowdhury N, Singer L: Health-related quality of life and 6 min walk distance in patients with idiopathic pulmonary fibrosis. Can Respir J 2011, 18:283-287.

39. Yorke J, Swigris J, Russell AM, Moosavi SH, Ng Man Kwong G, Longshaw M, Jones PW: Dyspnea-12 is a valid and reliable measure of breathlessness in patients with interstitial lung disease. Chest 2011, 139:159-164.

40. Zimmermann CS, Carvalho CR, Silveira KR, Yamaguti WP, Moderno EV, Salge JM, Kairalla RA, Carvalho CR: Comparison of two questionnaires which measure the health-related quality of life of idiopathic pulmonary fibrosis patients. Braz J Med Biol Res 2007, 40:179-187.

41. Idiopathic Pulmonary Fibrosis Clinical Research Network, Zisman DA Schwarz M, Anstrom KJ, Collard HR, Flaherty KR, Hunninghake GW: A controlled trial of sildenafil in advanced idiopathic pulmonary fibrosis. N Engl J Med 2010, 363:620-628.

42. Mahler DA, Weinberg DH, Wells CK, Feinstein AR: The measurement of dyspnea. Contents, interobserver agreement, and physiologic correlates of two new clinical indexes. Chest 1984, 85:751-758.

43. US Department of Health and Human Services (USDHHS): Guidance for Industry. In Patient-Reported Outcome Measures: Use in Medical Product Development to Support Labeling Claims. www.fda.gov/downloads/Drugs/ GuidanceComplianceRegulatoryInformation/Guidances/UCM193282.pdf.

44. Jones PW: Interpreting thresholds for a clinically significant change in health status in asthma and COPD. Eur Respir J 2002, 19:398-404.

45. Jones PW: St. George's Respiratory Questionnaire: MCID. COPD 2005, 2:75-79.

46. Jones PW, Beeh KM, Chapman KR, Decramer M, Mahler DA, Wedzicha JA: Minimal clinically important differences in pharmacological trials. Am 」 Respir Crit Care Med 2014, 189:250-255.

47. Kew KM, Mavergames C, Walters JA: Long-acting beta2-agonists for chronic obstructive pulmonary disease. Cochrane Database Syst Rev 2013 10, CD010177.

48. Cope S, Capkun-Niggli G, Gale R, Jardim JR, Jansen JP: Comparative efficacy of indacaterol $150 \mu \mathrm{g}$ and $300 \mu \mathrm{g}$ versus fixed-dose combinations of formoterol + budesonide or salmeterol + fluticasone for the treatment of chronic obstructive pulmonary disease-a network meta-analysis. Int J Chron Obstruct Pulmon Dis 2011, 6:329-344.

doi:10.1186/s12955-014-0124-

Cite this article as: Swigris et al:: The psychometric properties of the St George's Respiratory Questionnaire (SGRQ) in patients with idiopathic pulmonary fibrosis: a literature review. Health and Quality of Life Outcomes 2014 12:124

\section{Submit your next manuscript to BioMed Central and take full advantage of:}

- Convenient online submission

- Thorough peer review

- No space constraints or color figure charges

- Immediate publication on acceptance

- Inclusion in PubMed, CAS, Scopus and Google Scholar

- Research which is freely available for redistribution 